\title{
Erratum to: A Robust Tracking System for Low Frame Rate Video
}

\author{
Xiaoqin Zhang ${ }^{1}$ - Weiming $\mathrm{Hu}^{2} \cdot$ Nianhua $\mathrm{Xie}^{2}$ - Hujun $\mathrm{Bao}^{3}$. Stephen Maybank ${ }^{4}$
}

Published online: 17 July 2015

(C) Springer Science+Business Media New York 2015

\section{Erratum to: Int J Comput Vis \\ DOI 10.1007/s11263-015-0819-8}

The original article did not include the following grant information in the acknowledgements section:

This work is supported by NSFC (Grant Nos. 61472285, 6141101224, 61473212, 61100147, 61203241 and 61305035), Zhejiang Provincial Natural Science Foun- dation (Grants Nos. LY12F03016, LQ12F03004 and LQ13F030009), Project of science and technology plans of Zhejiang Province (Grants Nos. 2014C31062, 2015C31168).

The online version of the original article can be found under doi:10.1007/s11263-015-0819-8.

Xiaoqin Zhang

zhangxiaoqinnan@gmail.com

Weiming $\mathrm{Hu}$

wmhu@nlpr.ia.ac.cn

Nianhua Xie

nhxie@nlpr.ia.ac.cn

Hujun Bao

bao@cad.zju.edu.cn

Stephen Maybank

sjmaybank@dcs.bbk.ac.uk

1 Institute of Intelligent System and Decision, Wenzhou

University, Zhejiang, China

2 National Laboratory of Pattern Recognition, Institute of Automation, Chinese Academy of Sciences, Beijing, China

3 Department of Computer Science, Zhejiang University, Zhejiang, China

4 Department of Computer Science and Information Systems, Birkbeck College, London, UK 